EPJ Web of Conferences 81, 01021 (2014)

DOI: 10.1051/epjconf/ 20148101021

(C) Owned by the authors, published by EDP Sciences, 2014

\title{
Particle physics at the Pierre Auger Observatory
}

\author{
Jan $\mathrm{Ebr}^{1, a}$ \\ for the Pierre Auger Collaboration ${ }^{b}$ \\ ${ }^{1}$ Institute of Physics of the Czech Academy of Sciences, Na Slovance 1999/2, 18221 Praha 8
}

\begin{abstract}
The Pierre Auger Observatory is the largest detector of ultra-high energy cosmic rays (UHECR) in the world. These particles, presumably protons or heavier nuclei of energies up to $10^{20} \mathrm{eV}$, initiate extensive air showers which can be detected by sampling the particles that arrive at ground level or observing the fluorescence light generated during the passage of showers through the atmosphere - the Pierre Auger Observatory employs both these techniques. As the center-of-mass energies of the first interactions in the showers can be several orders of magnitude beyond the reach of the LHC, the UHECR provide an unique opportunity to study hadronic interactions. While the uncertainty in modeling these interactions is somewhat degenerate with the unknown composition of the primary beam, interaction models can be tested using data such as the depths of the maxima of the longitudinal development of the showers or their muon content. Particular sensitivity to interaction models is achieved when several observables are combined. Moreover, using careful data selection, proton-air cross section at the c.m.s. energy of 57 $\mathrm{TeV}$ per nucleon-nucleon pair can be obtained.
\end{abstract}

\section{Introduction}

The cosmic rays (CR), charged particles with extra-solar origin, are observed over more than ten decades in energies, between $10^{9} \mathrm{eV}$ and $10^{20} \mathrm{eV}$ in the Earth's laboratory system. Their energy spectrum is very steep - while the exact spectral index changes at several points of the spectrum, resulting in visible features, the spectrum generally follows the overall trend of roughly $E^{-3}$ across most of the energy range. The CR particles cannot be directly detected at the ground, because they interact in the upper levels of Earth's atmosphere and, while the lower energy CR are readily observed directly at balloon and satellite experiments, at energies above $10^{14} \mathrm{eV}$ the CR flux becomes so sparse that bringing a large enough instrument above the atmosphere for their detection becomes impractical. As often happens in physics, the hindrance of the CR interaction in the atmosphere can be turned into an advantage when the atmosphere itself is used as the detection volume and the detectors register phenomena caused by the interactions of the primary CR particles in it. Nevertheless, if an experiment aims to reach the upper part of the energy spectrum, it still needs to cover a sizeable area - at $10^{19} \mathrm{eV}$ the CR flux is of the order of 1 particle per square kilometer per year.

Above $10^{17} \mathrm{eV}$, the label ultra-high energy cosmic rays (UHECR) is often used. These are the most energetic particles in the Universe, carrying information on the most energetic astrophysical

\footnotetext{
a e-mail: ebr@fzu.cz

${ }^{b}$ Full author list: http://www.auger.org/archive/authors_2014_09.html
} 
processes. It is commonly assumed that their acceleration occurs in strong magnetic fields. However only very few objects are known to possess fields of the required intensity and spatial extend to explain the observed UHECR with energies over $10^{19} \mathrm{eV}$ - among these are active galaxy nuclei (AGN), relativistic jets and gamma ray bursts (GRB). A unique obstacle in identifying the sources of charged particles is that their trajectories are bent in the galactic and intergalactic magnetic fields so that most of the directional information is lost for all except the most energetic particles. Being magnetic processes, both the acceleration and deflection of the particles depend strongly on their electric charge - as most of the UHECR are believed to be either protons or nuclei of heavier elements (up to iron), the term mass (or even chemical) composition is frequently used to describe the charge distribution of the primary UHECR beam. As the UHECR are detected indirectly, modeling their interactions with the atmosphere is key to discerning the mass composition. This however requires us to understand hadronic interactions at center-of-mass energies up to hundreds of $\mathrm{TeV}$.

At the present time, the uncertainty in mass composition due to the extrapolation of hadronic interaction models from the LHC energy are the biggest obstacle for astrophysical interpretation of UHECR data. Again, where there is a complication, there is also an opportunity - namely to study the hadronic interactions at energies well beyond the reach of any accelerator in the foreseeable future.

\section{Extensive air showers}

When an UHECR particle interacts with an atmospheric nucleus, it initiates a cascade of many stages, eventually producing billions of secondary particles which form the so-called extensive air shower (EAS). Almost all particles produced in hadronic interactions are hadrons - most abundant are pions followed by other light mesons such as kaons and light baryons such as nucleons. Most of the hadrons created in the first interactions interact again with the notable exception of neutral pions which almost immediately decay into photons, giving rise to the electromagnetic cascade. Eventually, as the energy of individual particles gets lower after subsequent interactions, the charged pions and kaons also decay, producing a substantial number of muons. In the context of this meeting, it is noteworthy that most of the secondary interactions in an EAS are meson-nucleus interactions, often at very high energies (a $10^{19} \mathrm{eV}$ shower includes thousands of interactions of $\mathrm{TeV}$ mesons) and thus the description of the EAS is critically dependent on meson physics.

The development of the electromagnetic cascade, which contains most of the particles of the EAS, can be understood in terms of a simple model by Heitler [1] as a multiplicative process where in each step either a photon produces an electron-positron pair or an electron (positron) radiates a photon in the electromagnetic field of air molecules. As the splitting and radiation lengths in air are similar, we can assume them to be identical and have a value $\lambda_{r}$ : after $n$ such lengths, the shower has $2^{n}$ particles with typical energy of $E_{0} / 2^{n}$. When this energy falls bellow the critical energy $\left(E_{\text {crit }} \approx\right.$ $87 \mathrm{MeV})$ the multiplication stops and the shower maximum is reached at depth $X_{\max } \approx \lambda_{r} \ln \left(E / E_{\text {crit }}\right)$. Modeling the real EAS including hadrons is more complex [2] and requires the knowledge of their interactions - in the simplest approach at least of the depth of the first interaction $X_{0}$ and the average multiplicity of particle production $N$. If only pions are taken into account, $1 / 3$ of the energy goes to the electromagnetic cascade in each step and the shower development stops when the charged pions start decaying to muons. For a primary nucleus of atomic number $A$, a superposition model can be used, considering $A$ overlapping showers with energy $E_{0} / A$. The resulting estimate for depth of maximum of the shower is $X_{\max } \approx X_{0}+\lambda_{r}\left(\ln E_{0}-\ln N-\ln A+\ldots\right)$ meaning that heavier nuclei cause shallow showers at the same energy and that any departure from simple logarithmic dependence $X_{\max }$ on energy signifies a change in either mass composition or hadronic interactions.

In reality, the EAS are simulated using sophisticated Monte Carlo generators, but the main features observed in the phenomenological models are conserved. 
MESON 2014 $-13^{\text {th }}$ International Workshop on Production, Properties and Interaction of Mesons

\section{The Pierre Auger Observatory}

The Pierre Auger Observatory [3] located in Malargüe, Argentina is the largest UHECR detector in the world and uses surface and fluorescence detection techniques together in the hybrid mode. The Observatory consists of two parts - the surface detector (SD) [4] and the fluorescence detector (FD) [5] each with its own strengths and limitations. The SD comprises 1660 water Cherenkov detectors (WCD), each covering $10 \mathrm{~m}^{2}$, spread across $3000 \mathrm{~km}^{2}$ and samples the particles from the EAS that reach the ground level. It has a $100 \%$ duty cycle and well-known aperture. The WCD are arranged in a triangular grid with two different spacings: 1500 meters for most of the array and 750 meters for the smaller AMIGA area, reaching full sensitivity at $10^{18.5} \mathrm{eV}$ and $10^{17.5} \mathrm{eV}$ respectively. The arrival direction of the primary particle is reconstructed from the relative timing of the arrival of the secondary particles in different WCD and the energy is estimated using the absolute value of the observed signal reduced to a predefined distance from the shower core and zenith angle of the primary particle (the SD energy estimator).

The fluorescence detector consists of 27 telescopes at 4 sites along the edges of the SD array. These telescopes have a field of view of $28^{\circ} \times 30^{\circ}$ and observe the UV light from molecules of atmospheric nitrogen that are excited by the passage of the charged particles of the EAS. The duty cycle of the FD is only $13 \%$, but it allows not only the observation of the longitudinal profile of the shower in the air but also a very reliable energy measurement, which is almost calorimetric (barring the "invisible energy" carried by neutrinos and muons). Thus the showers observed by both FD and $\mathrm{SD}$ are used to calibrate the energy estimators of the latter. The systematic uncertainty on the energy scale is $14 \%$ and the energy resolution is $7-8 \%$ for the showers detected in the FD and $17-12 \%$ for showers detected only in the SD (outside of the FD duty cycle).

Additionally, the Observatory also includes various auxiliary devices, both for the detector calibration and atmospheric monitoring.

\section{Selected results}

\subsection{Fluorescence data}

As described in Sect. 2, the depth of the shower maximum $X_{\max }$ is sensitive to both the mass composition and hadronic interactions. While for each individual shower the $X_{\max }$ does not provide enough information to identify the primary particle unambiguously (even if we knew the exact properties of hadronic interactions), the distribution of depths of maxima of observed showers is related to the composition of the primary beam. To obtain this distribution [6], a fiducial volume selection is performed: first the range of values of $X_{\max }$ where most of the distribution lies is identified and then only such showers whose geometry would allow us to observe them should their $X_{\max }$ fall anywhere within this range are selected. From the distributions one can calculate the mean value and its fluctuations (corrected for detector resolution) - the dependence of the latter on the composition can also be understood within the superposition model. In the case of $A$ parallel showers, the combined fluctuations will be lower. The behavior of both variables suggests a change of composition from very light to heavier around $10^{18.5} \mathrm{eV}$. Moreover, we can observe that not all combinations of mean depth and fluctuations are compatible with predictions of hadronic interaction models - this fact is best understood when the data are interpreted in terms of mean primary mass and its fluctuations [7] as in Figure 1. In this Figure, three different hadronic interaction models are used: SIBYLL 2.1 [8], EPOS LHC [9] and QGSJET II-04 [10] and, while the last shows the largest tension, we must note that within the errors quoted, all three are compatible with the data. 

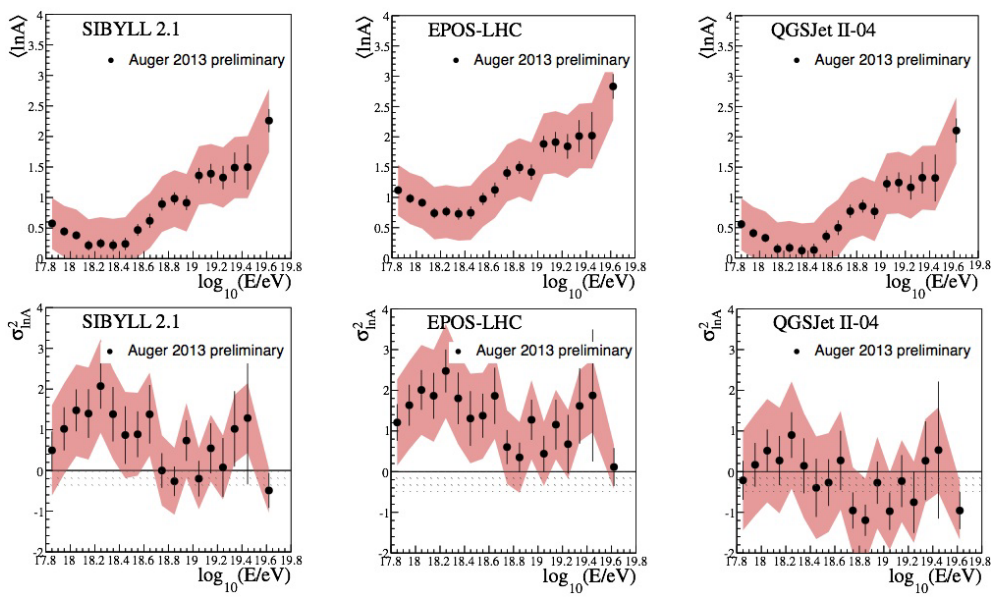

Figure 1. The mean depth of shower maximum and its fluctuations interpreted in terms of the logarithm of the mean mass of the primary beam and its fluctuations using three different hadronic interaction models [7].

In the energy range between $10^{18}-10^{18.5} \mathrm{eV}$, where the most data have been collected, the $X_{\max }$ results suggest at least a significant fraction of protons. Even if the precise composition is unknown, proton showers can be further selected by taking only the deepest part of the $X_{\max }$ distribution. It turns out that this part of the distribution has an exponential shape with a slope related to the mean free path of the first interaction and provides a relatively clean measurement of the proton-air particle-production cross-section [11]. The main systematics in this measurement arise from the slight dependence of the interpretation of the exponential slope on simulations and from possible photon and helium contamination in the data. Additionally, the total proton-proton crosssection can be calculated using Glauber theory with additional theoretical assumptions. The resulting value is $\sigma_{\mathrm{p}-\mathrm{p}}^{\mathrm{tot}}=133 \pm 13$ (stat) ${ }_{-20}^{+17}$ (syst) \pm 16 (Glauber) $\mathrm{mb}$ at the mean center-of-mass energy of $57 \pm 0.3$ (stat) \pm 6 (syst) TeV.

\subsection{Surface and combined data}

There are various observables that can be constructed using the signal in the WCD - the number of muons (at a given distance from the shower core) is easy to understand yet very sensitive to both the mass composition and hadronic interactions [12]. The superposition model predicts the heavier nuclei to produce more muons because there are less steps before the pions decay and thus a lower amount of energy is fed to the electromagnetic cascade in the form of neutral pions. The number of muons is influenced by many aspects of the hadronic interactions, such as mean multiplicity, inelasticity, baryon and strangeness production etc. The signal detected in the WCD is a combination of signals mainly from muons and electromagnetic (EM) particles. For highly inclined showers (zenith angle over $60^{\circ}$ ), the EM component is mostly absorbed in the air before reaching the ground and thus the ground signal is dominated by muons. For the rest of showers, the time structure of the signal can be analyzed using sophisticated methods such as smoothing or multivariate analysis to extract the muon information. All the methods indicate that the number of muons in data is higher than in simulations (Figure 2). Even though all data are compatible with simulation results within the systematic uncertainty when assuming pure iron composition, this assumption is not justified by the $X_{\max }$ results. 

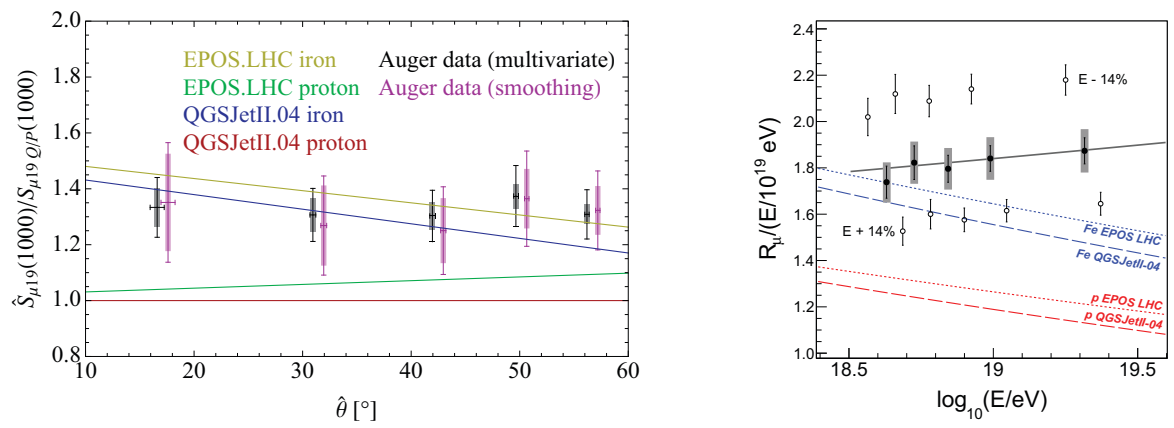

Figure 2. The number of muons deduced from vertical (left) [12] and highly inclined (right) [13] events. In both cases the number of muons is expressed relative to some reference value from simulations. Note that the data are presented as a function of zenith angle on the left panel, but as a function of energy on the right panel. The right panel also shows that shifting the energy scale by the systematic uncertainty makes the data essentially compatible with pure iron primary beam for both models used.

The interplay between SD and FD data can be further explored using showers that have been well recorded by both detectors [14]. For each observed event, showers are picked from a simulated library of either pure proton or mixed composition so that their longitudinal profiles show the best match with the observed one. Then the observed ground signal is compared with the ground signal predicted by the simulations for these matching showers. As the signal in general does not match, independent rescaling of the simulated electromagnetic and muon components is allowed in order to achieve an agreement. For an individual shower, rescaling the EM and muon components can be equally viable, but this degeneracy is broken if we require the same rescaling factors for all observed showers, as the components differ significantly in the zenith angle dependence of their attenuation. The results suggest that while the EM component is understood reasonably well, the muon component is underestimated in the simulations (Figure 3).

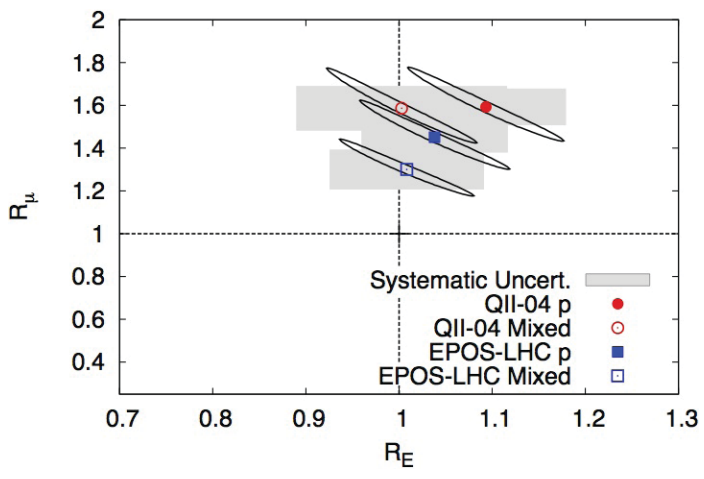

Figure 3. Results from the combined FD-SD analysis for the electromagnetic and muonic rescaling factors $R_{\mathrm{E}}$ and $R_{\mu}$ using two different hadronic interaction models with pure proton a mixed composition shower libraries. [14]

A new interesting approach which uses only SD data aims to measure the depth at which the muons arriving at ground were produced (muon production depth, MPD) [15]. Using showers with zenith angles between $55^{\circ}$ and $65^{\circ}$, the EM component is highly suppressed. Then, using timing information for individual muons from WCD between 1700 and 4000 meters from the shower core and geometry, the MPD distribution is reconstructed. The mean values of the maximum of this distribution, $X_{\max }^{\mu}$ can then be again interpreted in terms of mean primary mass using hadronic interaction models. The 


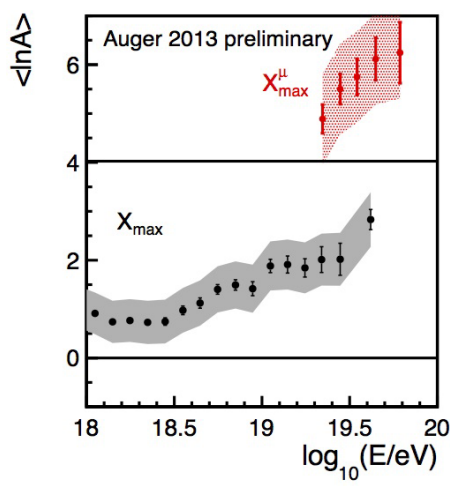

Figure 4. Comparison of the logarithm of the mean mass of the primary beam derived from $X_{\max }$ and $X_{\max }^{\mu}$ measurements using EPOS-LHC as the hadronic interaction model. [15]

results are compatible with the mass derived from $X_{\max }$ measurements when using QGSJET-II-04, but incompatible when using EPOS-LHC (Figure 4).

\section{Conclusions}

The Pierre Auger Observatory is sensitive to various observables related to hadronic interactions at extremely high energies. While most of these observables are also influenced by the (as of yet unknown) composition of the primary beam, useful information for the improvement of interaction models can be extracted, often from interplay between different observables, and a clean and easily interpreted result fo the proton-air cross-section can been extracted. Further progress is expected with more data, particularly thanks to the currently planned upgrade aimed at a more precise muon measurement.

\section{Acknowledgements}

We are grateful for the support by the grants of the Ministry of Education of the Czech Republic (MSMT-CR LG13007, 7AMB14AR005, CZ.1.05/2.1.00/03.0058) and the Czech Science Foundation grant 14-17501S.

\section{References}

[1] W. Heitler, The Quantum Theory of Radiation (1954)

[2] J. Matthews, Astroparticle Physics 22, 387-397 (2005)

[3] The Pierre Auger Collaboration, Nucl. Instrum. Meth. A523, 50 (2004).

[4] The Pierre Auger Collaboration, Nucl. Instrum. Meth. A613 29 (2010)

[5] The Pierre Auger Collaboration, Nucl. Instrum. Meth. A620 227 (2010)

[6] The Pierre Auger Collaboration, Phys. Rev. Lett. 104, 091101 (2010)

[7] The Pierre Auger Collaboration, JCAP 02026 (2013)

[8] E.-J. Ahn, R. Engel, T. K. Gaisser, P. Lipari, T. Stanev, Phys.Rev. D80, 094003 (2009)

[9] T. Pierog et al., EPOS LHC: test of collective hadronization with LHC data (2009)

[10] S. Ostapchenko, Phys. Rev. D83 014018 (2011)

[11] The Pierre Auger Collaboration, Phys. Rev. Lett. 109, 062002 (2012)

[12] B. Kegl [Pierre Auger Collaboration], Proc. of the 33rd ICRC (2013) arXiv:1307.5059

[13] I. Valino [Pierre Auger Collaboration], Proc. of the 33rd ICRC (2013) arXiv:1307.5059

[14] G. Farrar [Pierre Auger Collaboration], Proc. of the 33rd ICRC (2013) arXiv:1307.5059

[15] The Pierre Auger Collaboration, Phys. Rev. D90, 012012 (2014) 